\title{
Methane Production from Slaughterhouse Waste and Wheat Straw: Influence of Concentration
}

\section{Producción de Metano a Partir de Residuos de Matadero y Paja de Trigo: Influencia de la Concentración}

\author{
Meneses-Quelal W.O. and Velázquez-Martí B.
}

I International Congress of Science and Technology Morona Santiago-CICTMS 2020

Corresponding Author:

Meneses-Quelal W.O.

wasmeque@doctor.upv.es

borvemar@dmta.upv.es

Published: 29 August 2021

Production and Hosting by

Knowledge E

(c) Meneses-Quelal W.O. and Velázquez-Martí B.. This article is distributed under the terms of the Creative Commons Attribution License, which permits unrestricted use and redistribution provided that the original author and source are credited.

G OPEN ACCESS

Universitat Politècnica de Valencia. Escuela Técnica Superior de Ingeniería Agronómica y del Medio Natural. Departamento de Ingeniería Rural y Agroalimentaria. Valencia (España)

\section{Abstract}

The indiscriminate generation of slaughterhouse waste and agricultural waste can present pollution problems in the environment. An alternative to counteract these problems is the anaerobic digestion of waste through the production of biogas and methane as clean and renewable energy. In this sense, this study aimed to optimize methane production from anaerobic codigestion of slaughterhouse waste from cattle and wheat straw. The treatments were evaluated using anaerobic sludge as inoculum from the wastewater treatment plant of the city of Ibarra. The tests were carried out under mesophilic conditions $\left(38^{\circ} \mathrm{C}\right)$ in digesters with a useful volume of $186 \mathrm{ml}$. The influence of the substrate concentration was evaluated by anaerobically digesting 45 samples at different concentrations (5, 10 and $15 \mathrm{~g} \mathrm{VS/I)}$ with a substrate/inoculum ratio of 1:2. The highest accumulated methane yield occurred in the digesters composed of $15 \mathrm{~g} \mathrm{VS} / \mathrm{l}$. The maximum methane production was $320.48 \mathrm{Nml} / \mathrm{g} \mathrm{VS}$. The kinetics of the tests were adjusted with the cone model, where there were correlations greater than $99 \%$.

Keywords: biogas, methane, codigestion, synergy, inoculum, kinetics.

\section{Resumen}

La generación indiscriminada de residuos de matadero y desechos agrícolas pueden presentar problemas de contaminación en el medio ambiente. Una alternativa para contrarrestar estos problemas es la digestión anaeróbica de los desechos mediante la produción de biogás y metano como energía limpia y renovable. En este sentido el objetivo de este estudio es la optimización de la producción de metano a partir de la codigestión anaeróbica de residuos de matadero de ganado vacuno y paja de trigo. Los tratamientos se evaluaron empleando como inóculo lodo anaerobio de la planta de tratamiento de aguas residuales de la ciudad de Ibarra. Los ensayos se realizaron en condiciones mesofílicas $\left(38^{\circ} \mathrm{C}\right)$ en digestores de $186 \mathrm{ml}$ de volumen útil. La influencia de la concentración del sustrato se evaluó digiriendo anaeróbicamente 45 muestras a diferentes concentraciones (5, 10 y $15 \mathrm{~g} \mathrm{SV/I})$ con una relación sustrato/inóculo de 1:2. El mayor rendimiento acumulado de metano se produjo en los digestores compuestos por $15 \mathrm{~g}$ SV/I. La producción máxima de metano fue de $320,48 \mathrm{Nml} / \mathrm{g}$ SV. La cinética de los ensayos se ajustó con el modelo del cono, donde se tuvo correlaciones superiores al $99 \%$.

Palabras Clave: biogás, metano, codigestión, sinergia, inóculo, cinética. 


\section{Introducción}

La demanda creciente de carne por parte de la población mundial ha provocado un incremento de la contaminación ambiental. Esto ha hecho que se generen grandes cantidades de residuos en los mataderos o camales (RM) [1]. De esta manera, una alternativa para eliminar la presión ambiental generada por estos residuos es la digestión anaerobia de los residuos potencialmente fermentables [2]. El empleo de la digestión anaerobia (DA) resulta de mucha utilidad ya que es un proceso biológico que implica la degradación de materia orgánica y la producción de biogás con un alto porcentaje de metano [3]. Además, el biogás es una fuente de energía renovable y un factor clave para una futura sociedad independiente de los combustibles fósiles.

El alto contenido de grasas y proteínas, de los RM los convierte en sustratos atractivos para la producción de biogás [4]. Sin embargo, en el proceso de DA, estos residuos suelen acumular muchos ácidos grasos de cadena larga lo que hace difícil su degradación especialmente en el proceso de hidrólisis [5]. Como solución a este problema es recomendable realizar la codigestión de los RM con otro tipo de residuos, tales como los procedentes de las actividades agrícolas. El proceso de codigestión facilita la adquisición de un mejor equilibrio nutricional y disminuye la probabilidad de que el amoniaco y los lípidos inhiban el proceso [6].

El potencial de metano de los RM, pueden determinarse mediante una prueba tradicional de potencial de metano bioquímico (BMP). En los últimos años se ha publicado un elevado número de artículos científicos relacionados con las pruebas de BMP de una variedad de sustratos [7]. Sin embargo, los resultados de tales pruebas son variables y difíciles de comparar [8]. Los resultados variables se deben a diferencias tanto en la instrumentación como en los protocolos, así como a diferentes condiciones experimentales, especialmente la concentración del sustrato inicial [9].

La concentración de sustrato ha sido considerada como un factor importante que influye en la eficiencia del proceso de DA [10]. Sin embargo, hoy en día aún no está completamente claro la cantidad de sustrato ideal para conseguir una optimización completa del proceso de DA. A concentraciones muy bajas, existe el riesgo de que los microorganismos tengan una actividad metabólica baja y se produzcan cantidades muy bajas de biogás. Por el contrario, si la concentración de sustrato es demasiado alta, eso podría producir la acumulación de compuestos intermedios, lo que resulta la inhibición del proceso [11].

El propósito de esta investigación es evaluar la viabilidad y la eficiencia del proceso de digestión anaeróbica de los residuos de matadero en codigestión con la paja de trigo en condiciones mesofílicas, y a la vez evaluar el efecto de la concentración del sustrato en la producción de biogás. 


\section{Materiales y Métodos}

\subsection{Materia primas}

Como sustrato principal se empleó residuos de matadero de ganado vacuno (RM), los mismos que fueron recolectados del Camal Municipal de la ciudad de Guaranda (Ecuador). Los RM se recogieron de la salida de descarga del matadero del Camal Municipal. Las muestras recogidas consistieron en las aguas residuales del efluente del Camal, las mismas que estuvieron compuestas por sangre y despojos de los órganos internos generados en el sacrificio, junto con algo de estiércol. Como cosustrato, en cambio se empleó paja de trigo (PT). La paja se recogió de las granjas de la Universidad estatal de Bolívar, en la región de Guaranda. Las muestras se secaron bajo el sol, y posteriormente se refrigeraron hasta que empiece la puesta en marcha de los ensayos. En el laboratorio, para mantener la homogeneidad de las muestras, se molió la PT con un molino de laboratorio a un tamaño de partícula inferior a $3 \mathrm{~mm}$. La reducción del tamaño de las muestras se hizo con el fin de aumentar la superficie de las muestras a la actividad microbiana, ya que menor es el tamaño de partícula, mayor es la tasa de degradación de los residuos. Después de la molienda, la PT se almacenó a una temperatura inferior a $6^{\circ} \mathrm{C}$ hasta el momento de la incubación. De igual manera los RM fueron almacenados a la misma temperatura con fines de conservación.

Como inóculo (IN), para realizar las pruebas de BMP, se empleó lodo anaerobio mesofílico de aguas residuales. El inóculo fue recolectado de la planta de tratamiento de aguas residuales de la ciudad de Ibarra.

\subsection{Caracterización de la materia prima}

La caracterización de las muestras se realizó a través del análisis proximal de los datos. De esta manera, los sólidos totales (ST) y los sólidos volátiles (SV) se determinaron de acuerdo con las normas UNE-EN 18134 y UNE-EN ISO 18123, respectivamente. EI contenido de sólidos totales (TS) se determinó después de calentar $\left(105^{\circ} \mathrm{C}\right.$ durante 1 $\mathrm{hr}$ ), enfriar y desecar las muestras. Los SV se determinaron en un horno de mufla a una temperatura de $550^{\circ} \mathrm{C}$ mediante ignición de los residuos producidos en el análisis de ST a peso constante.

\subsection{Configuración experimental y producción de biogás}

Se realizaron pruebas por lotes para determinar las BMP de los sustratos individuales y de diversas combinaciones entre los residuos de matadero y los residuos de paja de trigo. Los ensayos se realizaron en botellas de vidrio con un volumen útil de $186 \mathrm{ml}$ y bajo condiciones mesofílicas $\left(T=38^{\circ} \mathrm{C}\right.$ ). Todas las botellas se cerraron con sellos de goma para mantener las condiciones anaerobias. Todos los experimentos se realizaron por triplicado y con una relación entre el sustrato y el inóculo (RSI) de 1:2.

Para evaluar las influencias de la concentración de sustrato en la prueba de BMP se varió la carga (sustrato + cosustrato) en tres concentraciones distintas: 5, 10 y 15 g SV/I. 
Para cada una de las concentraciones se estableció 5 relaciones entre el sustrato y el cosustrato (4:0, 3:1, 2;2, 1:3, y 0:4).

La producción de biogás se midió todos los días in situ, utilizando un analizador de biogás (BIOGÁS GA-5000 de Geotech). Las mediciones se realizaron durante 30 días en las que se caracterizó $\mathrm{H}_{2} \mathrm{~S}, \mathrm{CO}_{2}, \mathrm{CH}_{4}$ y $\mathrm{O}_{2}$. El fin de los experimentos se dio por terminado cuando la producción se estabilizó, es decir, cuando la producción diaria de biogás fue inferior al $1 \%$ de la producción acumulada. Finalmente, la producción acumulada de biogás se normalizó en condiciones estándar ( $P$ de $1 \mathrm{~atm}$ y $T$ de $25^{\circ} \mathrm{C}$ ) y se expresó en $\mathrm{ml}$ biogás/g SV.

\section{Resultados}

\subsection{Potenciales de metano de los residuos de matadero}

Los potenciales de metano obtenidos a diferentes concentraciones entre los RM y PT se presentan en la Figura 1. En ella se observa que la producción de $\mathrm{CH}_{4}$ en todos los biodigestores comenzó inmediatamente el primer día de digestión, después de lo cual comenzó a aumentar continuamente hasta el día 6 . Por el contrario, el rendimiento de $\mathrm{CH}_{4}$ comenzó a disminuir lentamente después del día 7, y a partir del día 20 la producción no fue significativa. A un TRH (tiempo de renención hidráulico) de 22 días y una concentración de $15 \mathrm{~g} \mathrm{SV} / \mathrm{l}$, el rendimiento máximo de $\mathrm{CH}_{4}$ fue de $320.48 \mathrm{Nml} / \mathrm{g}$ SV para una relacion sustrato/cosustrato de 4:0. Los ensayos demostraron que a mayor concentración de sustrato, la produccion de $\mathrm{CH}_{4}$ aumenta. Estos resultados están de acuerdo con el estudio de Raposo et al. [12], quien estudió la producción de metano del maíz para rangos de concentración de 5 a $15 \mathrm{~g} \mathrm{SV/I,} \mathrm{y} \mathrm{obtuvo} \mathrm{la} \mathrm{mayor} \mathrm{producción}$ de $\mathrm{CH}_{4}$ para $15 \mathrm{~g} \mathrm{SV/I}$. Muchos estudios revelan que el potencial de metano puede alcanzar asintóticamente un potencial máximo a concentraciones más altas [8].

Además, este estudio reveló que cuanto mayor es la concentracion de RM en los biodigestores, mayor es la producción de $\mathrm{CH}_{4}$. Esto es debido a que los RM contienen un alto contenido de grasas y proteínas, lo que los convierte en sustratos atractivos para la producción de metano [6].

En general, el análisis de varianza (ANOVA) de los rendimientos de metano demostró que para concentraciones de $5 \mathrm{~g} \mathrm{SV}, 10 \mathrm{~g}$ SV y $15 \mathrm{~g}$ SV, existen diferencias significativas $(p<0.05)$. El hecho de que haya diferencias de producción de metano pueden atribuirse a la acumulación de ácidos grasos volátiles (AGV) en algunos digestores. Además, las bajas concentraciones ( 5 y $10 \mathrm{~g} \mathrm{SV}$ ) pueden ser insuficientes para activar la actividad de los microorganismos causando estrés microbiano, disminución del pH y fallas en los digestores [13]. No obstante, los resultados obtenidos de este trabajo han hecho que la producción de metano obtenida refleje valores similares a otros sustratos previamente reportados por Cuetos et al. [14] y Bayr et al. [15], donde la producción de metano varío entre 400 a $700 \mathrm{NmL}$ de $\mathrm{CH}_{4} / \mathrm{g} \mathrm{SV}$, aunque las condiciones iniciales y parámetros de operación fueron diferentes. 


\section{Discusión}

Los resultados obtenidos en este trabajo sugieren que existe una correlación entre la concentración de sustrato y el potencial de metano en las pruebas de BMP. La norma alemana VDI 4630, 2006 [16] recomienda utilizar una concentración una concentración de sustrato de aproximadamente $10 \mathrm{~g} \mathrm{SV/I}$. Sin embargo, no existe una metodología estandarizada sobre la realización de pruebas de BMP que propongan requisitos exactos para la optimización de la producción de metano. En este sentido, existe el riesgo de que se utilicen concentraciones demasiado bajas y la DA no alcance la degradación completa o, por el contrario, se emplee concentraciones demasiado elevadas que inhiban el proceso de digestión. En este caso, se realizó el estudio con tres concentraciones una igual a $10 \mathrm{~g} \mathrm{SV/I}$, otra inferior a $10 \mathrm{~g} \mathrm{SV/I} \mathrm{y} \mathrm{otra} \mathrm{superior} \mathrm{a}$ $10 \mathrm{~g} \mathrm{SV/I}$. Los resultados revelaron que un aumento de la concentración aumenta el potencial metano, sin embargo, haría falta realizar más ensayos con concentraciones superiores a $15 \mathrm{~g}$ SV/I para determinar la asíntota de la curva y la estabilización de la producción de metano.

Según Wang et al. [8] un criterio sensato sería alcanzar $\geqslant 90 \%$ del potencial máximo de metano de los sustratos de biodegradados. No obstante, el proceso de digestión no es tan sencillo ya que depende de más factores como la relación sustrato/inóculo, temperatura, agitación, biodigestor, relación $\mathrm{C} / \mathrm{N}$ y tipo de materia prima.

En lo que se refiere a la sinergia del sustrato con el cosustrato los resultados no fueron tan alentadores ya que no mejoraron la producción de la monodigestión de los RM, aunque mejoraron notablemente la monodigestión de los residuos de PT. El hecho de que hubiese efectos antagónicos con los RM puede deberse a que en este estudio se realizó la codigestión de mezclas binarias. Baquerizo et al. [17] cuando se tiene mezclas de tres y cuatro sustratos se logran mayores efectos de sinergia que en mezclas de dos sustratos. Otro factor porque se presentó antagonismos se debe a que las mezclas binarias no han logrado proveer todos los nutrientes y elementos traza necesarios para que los microorganismos tengan una mayor actividad metanogénica [6].

La cinética de este ensayo reflejó claramente que el modelo del cono ajustó muy bien los datos experimentales con los pronosticados o predichos debido al fuerte respaldo del coeficiente de determinación $\left(R^{2}\right)$ muy cercano a 1. Además, el modelo del cono no sobrestimó los valores de BMP(t), ya que las diferencias porcentuales entre el potencial bioquímico de metano $(\mathrm{BMP}(\mathrm{t}))$ y el potencial bioquímico de metano máximo $\left(\mathrm{BMP}_{\max }\right)$ no fueron superiores al 10\% tal y como recomienda Zahan et al. [18]. El hecho que el modelo del cono haya proporcionado un buen ajuste se debe a la curva sigmoidea del modelo, ya que permite describir bien las fases de retardo, exponencial y estacionaria durante el proceso de digestión [19].

\section{Conclusiones}

Este estudio aborda una brecha significativa en el conocimiento sobre la la concentración de los sustratos en la pruebas de BMP y proporciona datos importantes sobre 
la sinergia y antagonismos en el proceso de codigestion anaerobia de los residuos de matadero con paja de trigo.

La concentración es un factor importante en esta investigación. El aumento de la concentración aumentó la producción de metano. Así, los digestores con concentraciones de $15 \mathrm{~g} \mathrm{SV/I}$ aumentaron su producción hasta $84 \%$ y $88 \%$ respecto de los digestores compuestos por 5 y g $10 \mathrm{~g} \mathrm{SV/I}$.

También, la codigestión mejoró la produccion de metano de la monodigestión de los residuos de paja de trigo en 7\%, 56\% y 48\% los ensayos de 5, 10 y 15 g SV/I respectivamente. El ajuste no lineal de la cinética, a través del modelo del cono, resultó muy eficaz. Se obtuvieron correlaciones superiores al 99\% entre los valores predichos y experimentales. Además, el modelo del cono no sobreestimó los valores de $\mathrm{BMP}_{\max }$ respecto de $\mathrm{BMP}(\mathrm{t})$, ya que se obtuvieron diferencias porcentuales del $10 \%$ aproximadamente.

Para obtener resultados más precisos, se recomienda que la concentración de sustrato no sea inferior a $10 \mathrm{~g} \mathrm{SV/I}$ y que las relaciones entre el sustrato e inóculo sean superiores a 1:2.

\section{Agradecimientos}

Este trabajo se ha realizado bajo la cooperación de la Universidad Estatal de Bolívar (Ecuador) y la Universidad Politécnica de Valencia (España).

\section{Conflicto de Intereses}

Declaramos que la presente investigación no presenta conflicto de intereses

\section{References}

[1] Sangodoyin AY, Agbawhe OM. Environmental study on surface and groundwater pollutants from abattoir effluents. Bioresour Technol. 1992;41(3):193-200.

[2] Aguilera ER. Generación de biogás mediante el proceso de digestión anaerobia, a partir del aprovechamiento de sustratos orgánicos. Rev Científica FAREM-Estelí. 2018;24:60-81.

[3] Cabbai V, Ballico M, Aneggi E, Goi D. BMP tests of source selected OFMSW to evaluate anaerobic codigestion with sewage sludge. Waste Manag. 2013;33(7):1626-32.

[4] Banks CJ, Wang Z. Development of a two-phase anaerobic digester for the treatment of mixed abattoir wastes. Water Sci Technol. 1999;40(1):69-76.

[5] Broughton MJ, Thiele JH, Birch EJ, Cohen A. Anaerobic batch digestion of sheep tallow. Water Res. 1998;32(5):1423-8.

[6] Pagés-Díaz J, Pereda-Reyes I, Taherzadeh MJ, Sárvári-Horváth I, Lundin M. Anaerobic co-digestion of solid slaughterhouse wastes with agro-residues: Synergistic and antagonistic interactions determined in batch digestion assays. Chem Eng J. 2014;245:89-98.

[7] Bauer A, Bösch P, Friedl A, Amon T. Analysis of methane potentials of steam-exploded wheat straw and estimation of energy yields of combined ethanol and methane production. J Biotechnol. 2009;142(1):505.

[8] Wang B, Strömberg S, Li C et al. Effects of substrate concentration on methane potential and degradation kinetics in batch anaerobic digestion. Bioresour Technol. 2015;194:240-6.

[9] Angelidaki I, Alves M, Bolzonella D, et al. Defining the biomethane potential (BMP) of solid organic wastes and energy crops: a proposed protocol for batch assays. Water Sci Technol. 2009;59(5):9-27. 
[10] Lianhua L, Dong L, Yongming S, Longlong M, Zhenhong $\mathrm{Y}$, Xiaoying K. Effect of temperature and solid concentration on anaerobic digestion of rice straw in South China. Int J Hydrogen Energy. 2010;35(13):7261-6.

[11] Elsayed M, Andres Y, Blel W, Gad A, Ahmed A. Effect of VS organic loads and buckwheat husk on methane production by anaerobic co-digestion of primary sludge and wheat straw. Energy Convers Manag. 2016;117:538-47.

[12] Raposo F, Banks CJ, Siegert I, Heaven S, Borja R. Influence of inoculum to substrate ratio on the biochemical methane potential of maize in batch tests. Process Biochem. 2006;41(6):1444-50.

[13] Wang YT, Suidan MT, Pfeffer JT, Najm I. Effects of some alkyl phenols on methanogenic degradation of phenol. Appl Environ Microbiol. 1988;54(5):1277-1279.

[14] Cuetos MJ, Gómez X, Otero M, Morán A. Anaerobic digestion of solid slaughterhouse waste (SHW) at laboratory scale: Influence of co-digestion with the organic fraction of municipal solid waste (OFMSW). Biochem Eng J. 2008;40(1):99-106.

[15] Bayr S, Rantanen M, Kaparaju P, Rintala J. Mesophilic and thermophilic anaerobic co-digestion of rendering plant and slaughterhouse wastes. Bioresour Technol. 2012;104:28-36.

[16] VDI VDI. 4630: Fermentation of organic materials-Characterisation of the substrate, sampling, collection of material data, fermentation tests. Verein Dtsch Ingenieure (VDI), Ed VDI Handb Energietechnik Berlin Beuth Verlag GmbH. 2006;44-59.

[17] Baquerizo CRJ, Díaz J, Pereda RI. El modelo de Buswell. Aplicación y comparación. Principales factores que influyen en su aplicación. Virtual Pro. 2016;168:19006241.

[18] Zahan Z, Othman MZ, Muster TH. Anaerobic digestion/co-digestion kinetic potentials of different agroindustrial wastes: A comparative batch study for C/N optimisation. Waste Manag. 2018;71:663-74.

[19] Li K, Liu R, Sun C. Comparison of anaerobic digestion characteristics and kinetics of four livestock manures with different substrate concentrations. Bioresour Technol. 2015;198:133-40.

[20] Alvarez R, Lidén G. Semi-continuous co-digestion of solid slaughterhouse waste, manure, and fruit and vegetable waste. Renew Energy. 2008;33(4):726-34.

[21] García-Gen S, Sousbie P, Rangaraj G et al. Kinetic modelling of anaerobic hydrolysis of solid wastes, including disintegration processes. Waste Manag. 2015;35:96-104.

[22] Jensen PD, Ge H, Batstone DJ. Assessing the role of biochemical methane potential tests in determining anaerobic degradability rate and extent. Water Sci Technol. 2011;64(4):880-6.

[23] Achinas S, Krooneman J, Euverink GJW. Enhanced biogas production from the anaerobic batch treatment of banana peels. Engineering. 2019;5(5):970-8.

[24] Mao C, Wang X, Xi J, Feng Y, Ren G. Linkage of kinetic parameters with process parameters and operational conditions during anaerobic digestion. Energy. 2017;135:352-60.

[25] Raposo F, Fernández-Cegrí V, De la Rubia MA et al. Biochemical methane potential (BMP) of solid organic substrates: evaluation of anaerobic biodegradability using data from an international interlaboratory study. J Chem Technol Biotechnol. 2011;86(8):1088-98. 